Article

\title{
The Reporting Frequency of Ketoacidosis Events with Dapagliflozin from the European Spontaneous Reporting System: The DAPA-KETO Study
}

\author{
Gabriella di Mauro 1,2,+, Annamaria Mascolo 1,2,*,+, Mario Gaio 1,2 1 , Concetta Rafaniello 1,2, \\ Antonella De Angelis ${ }^{2}$, Liberato Berrino ${ }^{2}$, Giuseppe Paolisso ${ }^{3,4}$, Francesco Rossi ${ }^{1,2, \ddagger}$ and Annalisa Capuano ${ }^{1,2, \ddagger}$
}

Citation: di Mauro, G.; Mascolo, A.; Gaio, M.; Rafaniello, C.; De Angelis,

A.; Berrino, L.; Paolisso, G.; Rossi, F.; Capuano, A. The Reporting

Frequency of Ketoacidosis Events with Dapagliflozin from the European Spontaneous Reporting System: The DAPA-KETO Study. Pharmaceuticals 2022, 15, 286. https://doi.org/10.3390/ ph15030286

Academic Editor: Réjean Couture

Received: 31 January 2022

Accepted: 23 February 2022

Published: 25 February 2022

Publisher's Note: MDPI stays neutral with regard to jurisdictional claims in published maps and institutional affiliations.

Copyright: (C) 2022 by the authors. Licensee MDPI, Basel, Switzerland. This article is an open access article distributed under the terms and conditions of the Creative Commons Attribution (CC BY) license (https:// creativecommons.org/licenses/by/ $4.0 /)$.
1 Campania Regional Centre for Pharmacovigilance and Pharmacoepidemiology, 80138 Naples, Italy; gabriella.dimauro@unicampania.it (G.d.M.); mario.gaio@unicampania.it (M.G.); concetta.rafaniello@unicampania.it (C.R.); francesco.rossi@unicampania.it (F.R.); annalisa.capuano@unicampania.it (A.C.)

2 Section of Pharmacology "L. Donatelli", Department of Experimental Medicine, University of Campania “Luigi Vanvitelli", 80138 Naples, Italy; antonella.deangelis@unicampania.it (A.D.A.); liberato.berrino@unicampania.it (L.B.)

3 Department of Advanced Medical and Surgical Sciences, University of Campania "Luigi Vanvitelli", 80138 Naples, Italy; giuseppe.paolisso@unicampania.it

4 Mediterrannea Cardiocentro, 80138 Naples, Italy

* Correspondence: annamaria.mascolo@unicampania.it; Tel.: +39-0815665805

+ Authors have contributed equally to this work and share first authorship.

$\ddagger$ Authors have contributed equally to this work and share lead authorship.

\begin{abstract}
Dapagliflozin was associated with an increased risk of diabetic ketoacidosis that has led to the European withdrawal of the authorization for the type 1 diabetes. However, it is still used for the treatment of type 2 diabetes. Therefore, we aim to evaluate the occurrence of dapagliflozininduced ketoacidosis events by using the European spontaneous reporting system. The reporting odds ratios (ROR) were computed to assess the reporting frequency of ketoacidosis events for dapagliflozin compared to Dipeptidyl peptidase-4 (DPP-4) inhibitors, insulins, or all other Sodiumglucose cotransporter-2 (SGLT-2) inhibitors. A total of 2406 cases with dapagliflozin reported at least one event of ketoacidosis. The three most reported events were: diabetic ketoacidosis $(1412 ; 55.39 \%)$, ketoacidosis $(476 ; 18.67 \%)$, and euglycaemic diabetic ketoacidosis $(296 ; 11.61 \%)$. Dapagliflozin was associated with the higher reporting frequency of ketoacidosis events compared to DPP-4 inhibitors (ROR 12.07, 95\%CI 11.67-13.81) or insulins (ROR 7.59, 95\%CI 7.13-7.89). A lower reporting frequency was instead observed compared to other SGLT2 inhibitors (ROR 0.91,95\%CI 0.87-0.96). Considering the higher reporting frequency of ketoacidosis observed with dapagliflozin then DPP-4 inhibitors or insulins, attention should be given to patients treated with this drug.
\end{abstract}

Keywords: dapagliflozin; ketoacidosis; diabetes mellitus; safety; adverse drug reaction

\section{Introduction}

Dapagliflozin is a selective, highly potent, reversible inhibitor of sodium-glucose cotransporter-2 (SGLT-2), which is responsible for $90 \%$ of glucose reabsorption. Through this mechanism, dapagliflozin increases urinary glucose excretion and reduces blood glucose levels [1-3]. It is indicated worldwide for the treatment of diabetes mellitus [4]. In particular, in the European Union (EU), it was initially authorized as monotherapy or as add-on combination therapy with other glucose-lowering agents for type 2 diabetes mellitus (T2DM), and lately, in 2019, for overweight patients with type 1 diabetes mellitus (T1DM) not sufficiently controlled with high doses of insulin. The add-on combination therapy is used when the HbA1c is not controlled by monotherapy [5]. Dapagliflozin has represented the first oral treatment approved for T1DM. This decision was based on the results of two 
Phase III clinical trials of the DEPICT clinical program (Dapagliflozin in Patients with Inadequately Controlled type 1 diabetes mellitus), which demonstrated that dapagliflozin associated with insulin significantly reduced glycated hemoglobin ( $\mathrm{HbA1c}$ ), weight, and total daily insulin dose in T1DM patients not adequately controlled [6]. However, since 25 October 2021, this last therapeutic indication was withdrawn by the manufacturing pharmaceutical industry in agreement with the European Medicine Agency (EMA) and the Health Products Regulatory Authority (HPRA) due to an increased risk of diabetic ketoacidosis observed in this subpopulation [7]. The risk of diabetic ketoacidosis was previously highlighted by clinical trials [8-11] and then by the Pharmacovigilance Risk Assessment Committee of EMA for the entire drug class of SGLT-2 inhibitors [12]. This risk is the reason that led the US Food and Drug Administration to reject the application of dapagliflozin for T1DM [13]. Diabetic ketoacidosis is a serious and rare complication caused by low insulin levels and high glucagon levels. Early signs and symptoms of diabetic ketoacidosis include polyuria, breathing difficulties, nausea, vomiting and anorexia, excessive thirst, abdominal pain, confusion, unusual asthenia, and sleepiness. More severe symptoms are dehydration, difficulty breathing, confusion, and coma [14-16]. Cases of this condition, including life-threatening ones, have occurred in patients taking SGLT2 inhibitors and a number of these cases have been atypical, with patients not having blood sugar levels as high as expected [12]. SGLT2 inhibitors induce ketoacidosis through multiple mechanisms. In the kidney, the inhibition of SGLT2 determines an increase in glycosuria and a reduction of lipolysis with a subsequent increase in ketone reabsorption and blood ketone levels. In the pancreas, as a result of glycosuria and through a direct action on pancreatic $\alpha$-cells, SGLT2 inhibitors determine an increase in glucagon levels, which induce an increase in hepatic lipolysis and ketogenesis $[17,18]$. Considering the seriousness of dapagliflozin-induced ketoacidosis and the recent authorization withdrawal for T1DM, we decided to perform a study to describe Individual Case Safety Reports (ICSRs) of ketoacidosis reported in the European database (Eudravigilance, EV) and to assess the reporting frequency of such cases with dapagliflozin.

\section{Results}

During the study period, 2406 out of 9225 ICSRs (26.08\%) with dapagliflozin as a suspected drug and reporting at least a ketoacidosis adverse event were retrieved from the $\mathrm{EV}$, of which $200(8.31 \%)$ referred to T1DM, 1191 (49.51\%) to T2DM, while in the remaining $42,18 \%$ (1015) of ICSRs the indication was not classifiable. The age ranged 18-64 years for all ICSRs (58.69\%) and each diabetes group (T1DM 59.00\%; T2DM 65.49\%; not classifiable $50.64 \%)$. Similarly, the gender most reported was feminine for all ICSRs $(55.11 \%)$ and for each diabetes group (T1DM 67.50\%; T2DM 52.31\%; not classifiable 55.96\%).The $98.79 \%$ of all ICSRs was classified as serious. In particular, the $98.50 \%$ for T1DM, the $98.57 \%$ for T2DM, and the $99.11 \%$ for not classifiable ICSRs. The main primary source was the healthcare professional for all ICSRs (94.31\%), T1DM (93.50\%), T2DM (95.55\%), and not classifiable $(93.00 \%)$. Most ICSRs reported one suspected drug $(85.99 \%)$ and no concomitant drugs (38.28\%). For T2DM, the $28.46 \%$ of ICSRs reported more than five concomitant drugs. Characteristics of ICSRs were presented in Table 1. From 2406 ICSRs, we observed a total of 2549 ketoacidosis adverse events (1.06 ADRs per ICSR) since more than one ADR could be reported in each ICSR. Specifically, $213(8.36 \%)$ ketoacidosis adverse events for T1DM, 1275 $(50.02 \%)$ for T2DM, and 1061 (41.62\%) for not classifiable ICSRs. The trend of ketoacidosis adverse events is shown in Figure S1. Most adverse events caused or prolonged hospitalization for all ketoacidosis PTs (50.33\%), ketoacidosis PTs in T1DM (46.95\%), ketoacidosis PTs in T2DM (52.08\%), and ketoacidosis PTs in not classifiable diabetes mellitus (48.92\%). The outcome of ketoacidosis adverse events was recovered/resolved for all ketoacidosis PTs (44.72\%), ketoacidosis PTs in T1DM (53.99\%), and ketoacidosis PTs in T2DM (51.14\%), while it was unknown for not classifiable diabetes mellitus (46.28\%). Seriousness and outcome criteria for all groups were presented in Table 2.The most reported PT was diabetic ketoacidosis $(1412 ; 55.39 \%)$, followed by ketoacidosis $(476 ; 18.67 \%)$, euglycaemic diabetic 
ketoacidosis $(296 ; 11.61 \%)$, metabolic acidosis $(115 ; 4.51 \%)$, ketonuria $(70 ; 2.75 \%)$, ketosis $(47 ; 1.84 \%)$, blood ketone body increased $(42 ; 1.65 \%)$, urine ketone body present $(30 ; 1.18 \%)$, acidosis $(23 ; 0.90 \%)$, diabetic ketosis $(20 ; 0.78 \%)$, diabetic ketoacidotic hyperglycemic coma $(6 ; 0.24 \%)$; blood ketone body present $(5 ; 0.20 \%)$, urine ketone body $(5 ; 0.20 \%)$, and blood ketone body $(2 ; 0.08 \%)$. The PTs specified for the type of diabetes mellitus are reported in Figure S2.

Table 1. Distribution for age, gender, seriousness, primary source, primary source country, number of suspected drugs, and number of concomitant drugs of Individual Case Safety Reports (ICSRs) reporting at least one event classifiable as ketoacidosis and having dapagliflozin as suspected drug among those reported in Eudravigilance from the date of marketing authorization to 11 September 2021.

\begin{tabular}{|c|c|c|c|c|}
\hline Variable & Level & $\begin{array}{l}\text { All ICSRs } \\
(n=2406)\end{array}$ & $\begin{array}{l}\text { ICSRs in T1DM } \\
\quad(n=200)\end{array}$ & $\begin{array}{l}\text { ICSRs in T2DM } \\
\quad(n=1191)\end{array}$ \\
\hline \multirow{4}{*}{ Age } & $<18$ years $(\%)$ & $8(0.33)$ & $8(4.00)$ & $0(0)$ \\
\hline & $18-64$ years $(\%)$ & $1412(58.69)$ & $118(59.00)$ & $780(65.49)$ \\
\hline & $>65$ years $(\%)$ & $443(18.41)$ & $17(8.50)$ & $233(19.56)$ \\
\hline & Missing (\%) & $543(22.57)$ & $57(28.50)$ & $178(14.95)$ \\
\hline \multirow{3}{*}{ Gender } & $\mathrm{F}(\%)$ & $1326(55.11)$ & $135(67.50)$ & $623(52.31)$ \\
\hline & $M(\%)$ & $1001(41.60)$ & $52(26.00)$ & $543(45.59)$ \\
\hline & Missing (\%) & $79(3.28)$ & $13(6.50)$ & $25(2.10)$ \\
\hline \multirow{2}{*}{ Seriousness of ICSR } & Serious (\%) & 2377 (98.79) & $197(98.50)$ & 1174 (98.57) \\
\hline & Not serious $(\%)$ & $29(1.21)$ & $3(1.50)$ & $17(1.43)$ \\
\hline \multirow{2}{*}{ Primary Source } & Healthcare Professional (\%) & $2269(94.31)$ & $187(93.50)$ & $1138(95.55)$ \\
\hline & Non-Healthcare Professional (\%) & $137(5.69)$ & $13(6.50)$ & $53(4.45)$ \\
\hline Primary Source Country for & European Economic Area (\%) & $1010(41.98)$ & $71(35.50)$ & $547(45.93)$ \\
\hline Regulatory Purposes & Non-European Economic Area (\%) & $1396(58.02)$ & $129(64.50)$ & $644(54.07)$ \\
\hline & $1(\%)$ & $2069(85,99)$ & $163(81.50)$ & $1015(85.22)$ \\
\hline & $2(\%)$ & $228(9.48)$ & $29(14.50)$ & $113(9.49)$ \\
\hline \multirow[t]{3}{*}{ Number of Suspected drug(s) } & $3(\%)$ & $72(2.99)$ & $6(3.00)$ & $39(3.27)$ \\
\hline & $4(\%)$ & $12(0.50)$ & $1(0.50)$ & $9(0.76)$ \\
\hline & $\geq 5(\%)$ & $25(1.04)$ & $1(0.50)$ & $15(1.26)$ \\
\hline \multirow{6}{*}{ Number of Concomitant drug(s) } & $0(\%)$ & $921(38.28)$ & $74(37.00)$ & $330(27.71)$ \\
\hline & $1(\%)$ & $301(12.51)$ & $47(23.50)$ & $138(11.59)$ \\
\hline & $2(\%)$ & $320(13.30)$ & $28(14.00)$ & $181(15.20)$ \\
\hline & $3(\%)$ & $211(8.77)$ & $20(10.00)$ & $116(9.74)$ \\
\hline & $4(\%)$ & $143(5.94)$ & $11(5.50)$ & $87(7.30)$ \\
\hline & $\geq 5(\%)$ & $510(21.20)$ & $20(10.00)$ & $339(28.46)$ \\
\hline
\end{tabular}

Table 2. Seriousness and outcome of ketoacidosis adverse events distributed by type of diabetes and having dapagliflozin as suspected drug among those reported in Eudravigilance from the date of marketing authorization to 11 September 2021.

\begin{tabular}{|c|c|c|c|c|}
\hline Variable & Level & $\begin{array}{l}\text { All ketoacidosis PT } \\
(n=2549)\end{array}$ & $\begin{array}{c}\text { Ketoacidosis PT } \\
\text { in T1DM } \\
(n=213)\end{array}$ & $\begin{array}{c}\text { Ketoacidosis PT } \\
\text { in T2DM } \\
(n=1275)\end{array}$ \\
\hline \multirow{6}{*}{ Seriousness } & Caused/Prolonged Hospitalization (\%) & $1283(50.33)$ & $100(46.95)$ & $664(52.08)$ \\
\hline & Other Medically Important Condition (\%) & $665(26.09)$ & $53(24.88)$ & $256(20.08)$ \\
\hline & Life Threatening (\%) & $510(20.01)$ & $50(23.47)$ & $304(23.84)$ \\
\hline & Results in Death (\%) & $45(1.77)$ & $6(2.82)$ & $23(1.80)$ \\
\hline & Not Serious (\%) & $35(1.37)$ & $4(1.88)$ & $22(1.73)$ \\
\hline & Disabling $(\%)$ & $11(0.43)$ & $0(0.00)$ & $6(0.47)$ \\
\hline \multirow{6}{*}{ Outcome } & Recovered/Resolved (\%) & $1140(44.72)$ & $115(53.99)$ & $652(51.14)$ \\
\hline & Unknown (\%) & $897(35.19)$ & $64(30.05)$ & $342(26.82)$ \\
\hline & Recovering/Resolving (\%) & $374(14.67)$ & $18(8.45)$ & $204(16.00)$ \\
\hline & Not Recovered/Not Resolved (\%) & $93(3.65)$ & $13(6.10)$ & $51(4.00)$ \\
\hline & Fatal (\%) & $30(1.18)$ & $2(0.94)$ & $17(1.33)$ \\
\hline & Recovered/Resolved With Sequelae (\%) & $15(0.59)$ & $1(0.47)$ & $9(0.71)$ \\
\hline
\end{tabular}




\subsection{ROR in General Diabetes}

In the analysis considering all ICSRs, dapagliflozin was associated with an increased reporting frequency of ketoacidosis adverse events compared to DPP-4is (ROR 12.07, 95\%CI 11.67-13.81; Figure 1A) or insulin (ROR 7.59, 95\%CI 7.13-7.89; Figure 1B), while a lower reporting frequency was observed compared to other SGLT2 inhibitors (ROR 0.91, 95\%CI 0.87-0.96; Figure 1C). The RORs computed for each specific PT are reported in Figure 1 for all comparisons.

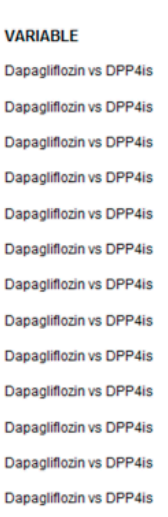

Dapaglitiozin vs DPP 4is



ROR
29.93
4.75
37.11
34.35
3.21
25.18
16.05
33.75
24.09
75.08
6.42
5.35
12.70

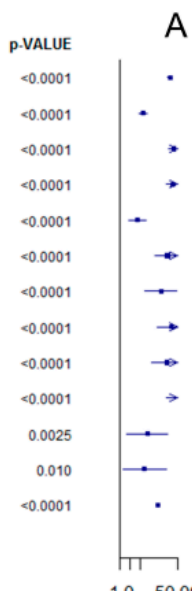

$1.0 \quad 50.00$

B

VARIABLE

Dapagilifozin vs Insulin

Dapaglifozin vs Insulin

Dapagliflozin vs Insulin

Dapaglifiozin vs Insulin

Dapagifitozin vs Insulin

Dapaglifozin vs Insulin

Dapagifitozin vs Insulin

Dapagititozin vs Insulin

Dapaglifozin vs Insulin

Dapagliflozin vs Insulin

Dapaglifozin vs Insulin

Dapaglifozin vs insulin

Dapagilifozin vs insulin

Dapagliflozin vs Insulin

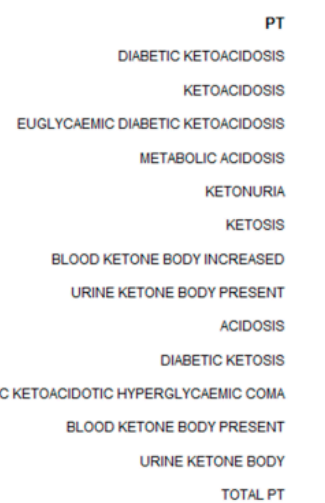

15506

\begin{tabular}{r}
$95 \% \mathrm{Cl}$ \\
$5.41-6.16$ \\
$9.46-12.12$ \\
\hline $02.01-238.43$ \\
$12.55-22.09$ \\
$13.64-29.32$ \\
$5.87-12.41$ \\
$3.26-6.66$ \\
$3.39-7.99$ \\
$3.78-10.36$ \\
\hline $2.61-7.26$ \\
$1.00-5.45$ \\
$0.10-0.56$ \\
\hline $3.46-41.33$ \\
$7.13-7.89$
\end{tabular}

p.value

$<0.0001$

$<0.0001$

$<0.0001$
$<0.0001$

$<0.0001$

$<0.0001$

$<0.0001$

$<0.0001$

$<0.0001$

$<0.0001$

$<0.0001$

0.0441

0.0004

$<0.0001$

$<0.0001$

VARIABLE

Dapagilinozin vs Other SGLT2is

Dapagliffozin vs Other SGLT2is

Dapaglifiozin vs Other SGLT2is

Dapaglifozin vs Other SGLT2is

Dapagififozin vs Other SGLT2is

Dapaglifozin vs Other SGLT2is

Dapagliffozin vs Other SGLT2is

Dapaglifozin vs Other SGLT2is

Dapaglifozin vs Other SGLT2is

Dapagilinozin vs Other SGLT2is

Dapagififozin vs Other SGLT2is

Dapaglifiozin vs Other SGLT2is

Dapaglifinozin vs Other SGLT2is

Dapagliffozin vs Other SGLT2is

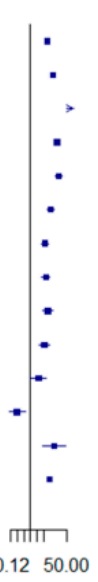

C

p.VAlUE
$<.0001$
0.0372
0.2830
$<.0001$
0.0422
0.0263
0.0182
0.3103
0.2592
0.0297
0.3454
0.7399
0.3707
0.0002

Figure 1. ROR of ketoacidosis adverse events for dapagliflozin compared to DPP-4is (A), insulin (B), or other SGLT2 inhibitors (C) in all cases of diabetes mellitus. 
In the direct comparison between SGLT2 inhibitors, no difference was observed when dapagliflozin was compared to canagliflozin (ROR 1.04, 95\%CI 0.99-1.10; Figure S3) and ertugliflozin (ROR 1.48, 95\%CI 0.95-2.31; Figure S4). On the contrary, a lower reporting frequency was observed when dapagliflozin was compared to empagliflozin (ROR 0.77, 95\%CI 0.73-0.82; Figure S5). The RORs related to direct comparisons computed for each specific PT are reported in Figures S3-S5.

\section{2. $R O R$ in T1DM}

In the analysis considering only T1DM ICSRs, dapagliflozin was associated with an increased reporting frequency of ketoacidosis adverse events compared to insulin (ROR 9.60, 95\%CI 8.13-11.32; Figure 2A), while no difference was observed compared to other SGLT2 inhibitors (ROR 1.11, 95\%CI 0.92-1.35; Figure 2B). The RORs computed for each specific PT are reported in Figure 2 for all comparisons.



Figure 2. ROR of ketoacidosis adverse events for dapagliflozin compared to insulin (A) or other SGLT2 inhibitors (B) in T1DM.

In the direct comparison between SGLT2 inhibitors, no difference was observed when dapagliflozin was compared to empagliflozin (ROR 0.91, 95\%CI 0.72-1.15; Figure S6), while a lower reporting frequency was observed when dapagliflozin was compared to canagliflozin (ROR 1.26, 95\%CI 1.02-1.55; Figure S7). 
In the analysis comparing dapagliflozin in T1DM vs. dapagliflozin in T2DM, the reporting frequency was increased of 3.36-fold in T1DM (95\%CI 2.84-3.98; Figure 3). The RORs computed for each specific PT are reported in Figure 3.

\begin{tabular}{|c|c|c|c|c|c|}
\hline VARIABLE & PT & ROR & $95 \% \mathrm{Cl}$ & p-VALUE & \\
\hline Dapagliflozin in T1DM vs Dapagliflozin in T2DM & DIABETIC KETOACIDOSIS & 3.16 & 2.56-3.91 & $<0.0001$ & - \\
\hline Dapagliflozin in T1DM vs Dapagliflozin in T2DM & KETOACIDOSIS & 4.27 & 3.14-5.82 & $<0.0001$ & - \\
\hline Dapagliflozin in T1DM vs Dapagliflozin in T2DM & EUGLYCAEMIC DIABETIC KETOACIDOSIS & 1.52 & $0.92-2.52$ & 0,0998 & - \\
\hline Dapagliflozin in T1DM vs Dapagliflozin in T2DM & KETONURIA & 1.63 & $0.65-4.11$ & 0,2921 & $=-$ \\
\hline Dapagliflozin in T1DM vs Dapagliflozin in T2DM & KETOSIS & 3.21 & $1.23-8.41$ & 0,0121 & $=$ \\
\hline Dapagliflozin in T1DM vs Dapagliflozin in T2DM & BLOOD KETONE BODY INCREASED & 5.74 & $2.06-15.97$ & 0,0002 & $\because-$ \\
\hline Dapagliflozin in T1DM vs Dapagliflozin in T2DM & TOTAL PT & 3.36 & 2.84-3.98 & $<0.0001$ & - \\
\hline
\end{tabular}

Figure 3. ROR of ketoacidosis adverse events for dapagliflozin in T1DM compared to dapagliflozin in T2DM.

\section{Discussion}

The present study evaluated the effect of dapagliflozin on ketoacidosis adverse events by using data from the EV database. Our main result reflects a higher reporting frequency of ketoacidosis adverse events with dapagliflozin, supporting in part the risk observed in other observational studies [19-21]. However, data reported in the EV database concerns suspected ADRs that have been observed following the use of a drug, but which are not necessarily related to or caused by the drug.

In our general descriptive analysis, we found that about $26 \%$ of all ICSRs of dapagliflozin reported at least one ketoacidosis adverse event and that most of them occurred in T2DM patients (49.51\%). This result is in line with the most recent marketing authorization of dapagliflozin for T2DM that happened about 7 years earlier than that of T1DM. According to our results, ketoacidosis adverse events occurred most frequently in female patients, enough to be considered among risk factors for diabetic ketoacidosis [22]. Lastly, it should be noted that women are more susceptible to experience ADRs due to hormones and changes in pharmacokinetics, independently from the drug class [23-25]. Moreover, we observed most ICSRs in patients aged 18-64 years in line with marketing authorization details that stated the use only in adult patients [26]. However, results in the literature are conflicting and an observational study found that age and sex did not modify the association of dapagliflozin and ketoacidosis [19].

The $94.31 \%$ of ICSRs were reported by HCPs in accordance with other studies [27-31]. In most ICSRs was reported one suspected drug and no concomitant drugs. This may imply a low presence of contributing factors to the occurrence of adverse events, but the incompleteness of information should be considered as well.

Most ICSRs were classified as serious due to hospitalization and because the most reported events (such as diabetic ketoacidosis, ketoacidosis, and euglycaemic diabetic ketoacidosis) are included in the Important Medical Event (IME) List 24.1 of EMA. Moreover, ketoacidosis adverse events result in a complete resolution in most ICSRs despite a little more of $30 \%$ had an unknown outcome that could affect the proportion of cases with a complete resolution that, therefore, cannot be perfectly estimated. Accordingly, in the literature, patients are used to restart the treatment with SGLT2 inhibitors following the resolution of the event [32]. However, we could not establish with our data if the treatment 
was restarted again due to our study limitations. Knowing the outcome is also important to better understand the impact of the higher reporting frequency of ketoacidosis events on the patients' quality of life.

In the analysis related to all diabetes ICSRs, dapagliflozin was associated with a 12.7and 7.6-fold increased reporting frequency of ketoacidosis adverse events compared to DPP4is and insulin, respectively. Considering literature data, previous studies on spontaneous reporting system also found a high reporting frequency of ketoacidosis associated with SGLT2 inhibitors $[33,34]$. However, when the risk of ketoacidosis was evaluated in terms of hazard, observational studies found lower estimates. Specifically, a population-based cohort study comparing patients with T2DM who are new users of SGLT-2 inhibitors with those receiving DPP-4is showed a Hazard Ratio of 2.85 (95\%CI 1.99-4.08) [19]. Moreover, a nationwide register-based cohort study from two countries found that the use of SGLT2 inhibitors compared with Glucagon Like Peptide-1 receptor agonists was associated with a 2.1-fold increased risk of diabetic ketoacidosis (95\%CI 1.01-4.52) in T2DM patients [21]. Furthermore, a meta-analysis of randomized trials and cohort studies found a $2.5-(95 \% \mathrm{CI}$ 1.16-5.21) and 1.7-fold (95\%CI 1.07-2.83) higher risk of diabetic ketoacidosis with SGLT2 inhibitors compared with reference groups, respectively [35]. These differences in the estimates may be due to the different study design and analysis.

Dapagliflozin used for T1DM was associated with a 3.4-fold increased reporting frequency of ketoacidosis adverse events compared with dapagliflozin used for T2DM. In the analysis considering only T1DM ICSRs, we found a reporting frequency of ketoacidosis adverse events for dapagliflozin compared to insulins that was higher than that observed in all diabetes cases. Indeed, it was increased 9.6-fold compared to 7.6-fold observed in all cases. The increased frequency observed in T1DM can be explained by the renal and pancreatic effects of SGLT2-inhibitors that are emphasized in patients in add-on combination therapy with insulin, since to minimize the risk of hypoglycemia it is necessary to decrease the insulin dose. This furtherly alters the glucagon/insulin ratio contributing to an additional increase in circulating ketone body levels [36]. This mechanism is probably exploit for their use in heart failure [37], since ketones can be cardiac substrates that improve the metabolic efficiency of the heart [4]. Furthermore, it should be considered that diabetic ketoacidosis is caused by low levels of insulin and that the administration of intravenous insulin is frequently used to resolve this adverse event [38]. Indeed, to prevent the potentially dangerous complications associated with diabetes ketoacidosis, patients in treatment with SGLT2 inhibitors who develop the event should discontinue the medication, undergo ketone evaluation, and start insulin therapy, if blood ketones are detected [39]. The risk in T1DM was assessed in previous clinical trials. The Dapagliflozin Evaluation in Patients with Inadequately Controlled T1DM (DEPICT-2) trial found that diabetic ketoacidosis was more reported in the dapagliflozin groups compared to placebo with the majority of events that were mild or moderate in severity and that resolved after treatment [11]. Similarly, the pooled analysis of DEPICT-1 and DEPICT-2 studies found an increased onset of diabetic ketoacidosis with dapagliflozin compared with placebo over 52 weeks [40]. However, a recent meta-analysis of randomized controlled clinical trials seem to find that the short-term use ( 24 weeks) of dapagliflozin associated with insulin for T1DM was not associated with an increased risk of diabetic ketoacidosis, but as highlighted by Authors additional high-quality studies are needed to determine its long-term safety [41]. However, the risk of ketoacidosis associated SGLT2 inhibitors has led over the years to recommendations for the management of this event in patients with T1DM [42]. Finally, a lower reporting frequency was observed when dapagliflozin was compared to other SGLT2 inhibitors in all diabetes cases, while no difference was showed in T1DM cases. This result may highlight that these drugs share the same mechanism of action suggesting a drug class effect [19]. Indeed, cases of ketoacidosis have been also reported for the other SGLT2 inhibitors [43-45]. Moreover, this may influence the choice of a drug from this therapeutic class but further studies are needed to confirm this hypothesis. 


\section{Strengths and Limitations}

Our study has some strengths and limitations. Among the strengths, we should consider that the spontaneous reporting system is a useful and inexpensive tool for the collection and analysis of drugs safety data and the better characterization of the drug safety profiles. Indeed, through data from the spontaneous reporting system, we can detect ADRs not detectable during the pre-marketing phase, including rare and serious ones. Therefore, through the EV database, we have analyzed a large amount of ICSRs related to dapagliflozin and the entire European area. Among limitations, there is the underreporting and the poor quality of information listed in each ICSR. In particular, underreporting is a major limitation of spontaneous reporting systems that reduces sensitivity because it underestimates the frequency and the impact of a given ADRs. Moreover, the incompleteness of information reported in the ICSRs could have affected our results by influencing the case selection based on the types of diabetes mellitus. Another limitation of our study is that we cannot establish causality with our data. Finally, an important specific limitation for the SGLT2 inhibitors as well as for other glucose-lowering therapies with cardio-metabolic benefits is related to the barriers of prescribing such as costs and unfamiliarity with their use that could have limited study results [46-48].

\section{Materials and Methods}

\subsection{Study Design}

The DAPAgliflozin and KETOacidosis (DAPA-KETO) study is a systematic analysis of the European pharmacovigilance database (EudraVigilance).

\subsection{Data Source}

Data on ICSRs with dapagliflozin as a suspected drug were retrieved from the website of suspected adverse drug reactions (ADRs) of EV (www.adrreports.eu, accessed on 21 September 2021). The EV is supervised by the EMA and it is a system used for the management and analyses of ICSRs related to both medicines or vaccines, which are authorized or are being evaluated in clinical trials within the European Economic Area (EEA). The EV contains all ICSRs reported by a healthcare professional (HCP) or a non-HCP to an EU national competent authority or a marketing authorization holder. These data are publicly available for transparency through the EMA website (www.adrreports.eu, accessed on 21 September 2021).

\subsection{ICSRs Selection with Line Listing}

By using the line listing function, ICSRs reporting dapagliflozin as a suspected drug were retrieved from the date of marketing authorization granted by the EMA to 11 September 2021. According to the Assessment Report of SGLT2 inhibitors published by the EMA [49], we identified a case of ketoacidosis by using the following preferred terms (PTs) of the Medical Dictionary for Regulatory Activities (MedDRA): acidosis, blood ketone body present, blood ketone body increased, blood ketone body, diabetic ketoacidosis, diabetic ketoacidotic hyperglycemic coma, diabetic ketosis, euglycaemic diabetic ketoacidosis, ketoacidosis, ketonuria, ketosis, metabolic acidosis, urine ketone body present, and urine ketone body. MedDRA terminology is described elsewhere [29,50].

\subsection{Descriptive Analyses}

Information on patient characteristics (age and gender), the seriousness of the adverse event, primary source qualification, primary source country for regulatory purposes, number of suspected drugs other than dapagliflozin, and number of concomitant drugs were provided for all ICSRs. ICSRs were also classified and summarized based on the therapeutic indication in those related to T1DM or T2DM. If the dapagliflozin therapeutic indication was not specific, the ICSRs were defined as not classifiable for the type of diabetes and described separately. 
In accordance with the International Council on Harmonization E2D guidelines, we classified the seriousness of a ketoacidosis adverse event as life-threatening, results in death, caused/prolonged hospitalization, disabling, determines a congenital anomaly/birth defect, or other medically important condition. If more than one criterion of seriousness was reported for an adverse event, we chose for classification the most serious. The outcome of a ketoacidosis adverse event was classified as "Recovered/Resolved", "Recovering/Resolving", "Recovered/Resolved With Sequelae", "Not Recovered/Not Resolved", "Fatal", and "Unknown. The outcome with the lower level of resolution was chosen for classification whether an adverse event of ketoacidosis reported two or more different outcomes. Ketoacidosis adverse events of dapagliflozin were summarized for each type of diabetes.

\subsection{Descriptive Analyses}

The Reporting Odds Ratio (ROR) and its' 95\% confidence interval (95\%CI) was computed to assess the reporting frequency of ketoacidosis adverse events for dapagliflozin compared to dipeptidyl peptidase- 4 inhibitors (DPP-4is) or insulins. We chose DPP4is as the comparator group because they are a second-line treatment similarly used for diabetes and have no known association with diabetic ketoacidosis [51]. While we used insulins because they are widely used for T1DM.

Moreover, a sub-analysis was performed to compare dapagliflozin with all other SGLT2 inhibitors, and with each specific SGLT2 inhibitor. Single drugs considered in reference groups are listed in Table S1. For drugs used in both diabetes forms, analyses were performed on all ICSRs retrieved from the EV website and separately for those classified as T1DM. Finally, the ROR was computed to evaluate the reporting frequency of ketoacidosis adverse events with dapagliflozin used in T1DM compared to its use in T2DM. RORs were carried out for PTs reported at least in a number of cases $\geq 3$, and also for the sum of all PTs of ketoacidosis (Total PT). Data management and analysis were performed with Microsoft Excel 2019 and R (version 3.2.2, R Development Core Team). Forest plots were performed using R (version 3.2.2, R Development Core Team).

\section{Conclusions}

We carried out descriptive and statistical analyses from 2406 ICSRs (covering 2549 ketoacidosis ADRs) related to dapagliflozin. Ketoacidosis ADRs were serious in more than $98 \%$ of cases and the outcome was resolved in $44 \%$ of cases. The most reported ketoacidosis adverse events were diabetic ketoacidosis, ketoacidosis, and euglycaemic diabetic ketoacidosis. Applying the ROR, we found that dapagliflozin was associated with a higher reporting frequency of ketoacidosis when compared to DPP4is or insulin in all analyses. No difference was instead observed in the comparison with all other SGLT2 inhibitors for all analyses. The higher risk of ketoacidosis with dapagliflozin has led to the marketing authorization withdrawal for T1DM. However, since dapagliflozin remains authorized for T2DM and that the risk of ketoacidosis can be associated with all SGLT2 inhibitors, attention should be given to patients treated with these drugs. Indeed, patients in therapy with SGLT2 inhibitors must contact their medical doctors if they have symptoms such as rapid weight loss, nausea or vomiting, stomach pain, excessive thirst, fast and deep breathing, confusion, unusual sleepiness, and others. Moreover, the occurrence of this adverse event in the setting of relative euglycemia could be critical for recognizing this life-threatening diabetes complication. Ketoacidosis is being increasingly reported with SGLT2 inhibitors since these drugs are prescribed in the primary care setting. In conclusion, given the potential seriousness of dapagliflozin-induced ketoacidosis and considering the intrinsic limitations of our study, we believe that further high-quality clinical studies should be conducted on this topic, especially in T2DM patients, to better estimate the impact of SGLT2 inhibitors on diabetic ketoacidosis. 


\begin{abstract}
Supplementary Materials: The following supporting information can be downloaded at: https: / / www.mdpi.com/article/10.3390/ph15030286/s1, Table S1: Drugs included in reference groups; Figure S1: Trend of ketoacidosis adverse events for each type of diabetes mellitus; Figure S2: ketoacidosis adverse events for type of diabetes mellitus; Figure S3: ROR of ICSRs with ketoacidosis adverse events for dapagliflozin compared to canagliflozin in all diabetes mellitus cases; Figure S4: ROR of ICSRs with ketoacidosis adverse events for dapagliflozin compared to ertugliflozin in all diabetes mellitus cases; Figure S5: ROR of ICSRs with ketoacidosis adverse events for dapagliflozin compared to empagliflozin in all diabetes mellitus cases; Figure S6: ROR of ICSRs with ketoacidosis adverse events for dapagliflozin compared to empagliflozin in T1DM; Figure S7: ROR of ICSRs with ketoacidosis adverse events for dapagliflozin compared to canagliflozin in T1DM.
\end{abstract}

Author Contributions: Conceptualization, F.R. and A.C.; Data curation, G.d.M., A.M. and M.G.; Formal analysis, G.d.M. and A.M.; Investigation, C.R.; Methodology, G.d.M., A.M., C.R., L.B. and A.C.; Supervision, F.R. and A.C.; Validation, L.B. and G.P.; Visualization, A.D.A., G.P. and F.R.; Writing—original draft, G.d.M. and A.M.; Writing—review \& editing, A.D.A., L.B., G.P., F.R. and A.C. All authors have read and agreed to the published version of the manuscript.

Funding: This research received no external funding.

Institutional Review Board Statement: Not applicable.

Informed Consent Statement: Not applicable.

Data Availability Statement: European Pharmacovigilance data are available at www.adrreports.eu (accessed on 21 September 2021).

Conflicts of Interest: The authors declare no conflict of interest.

\title{
References
}

1. Plosker, G.L. Dapagliflozin: A Review of Its Use in Patients with Type 2 Diabetes. Drugs 2014, 74, 2191-2209. [CrossRef] [PubMed]

2. Vivian, E.M. Dapagliflozin: A new sodium-glucose cotransporter 2 inhibitor for treatment of type 2 diabetes. Am. J. Health Pharm. 2015, 72, 361-372. [CrossRef] [PubMed]

3. Balakumar, P.; Sundram, K.; Dhanaraj, S.A. Dapagliflozin: Glucuretic action and beyond. Pharmacol. Res. 2014, 82, 34-39. [CrossRef] [PubMed]

4. Mascolo, A.; Scavone, C.; Scisciola, L.; Chiodini, P.; Capuano, A.; Paolisso, G. SGLT-2 inhibitors reduce the risk of cerebrovascular/cardiovascular outcomes and mortality: A systematic review and meta-analysis of retrospective cohort studies. Pharmacol. Res. 2021, 172, 105836. [CrossRef]

5. National Institute for Health and Care Excellence (NICE). Type 2 Diabetes in Adults: Management. NICE Guideline. Available online: https:/ / www.nice.org.uk/guidance/ng28 (accessed on 17 February 2022).

6. Paik, J.; Blair, H.A. Dapagliflozin: A Review in Type 1 Diabetes. Drugs 2019, 79, 1877-1884. [CrossRef]

7. HPRA. Forxiga (Dapagliflozin) $5 \mathrm{mg}$ Should No Longer Be Used for the Treatment of Type 1 Diabetes Mellitus. Available online: https:/ / www.hpra.ie/docs/default-source/default-document-library/important-safety-information-forxiga-(dapagliflozin) -5mg.pdf?sfvrsn=0 (accessed on 3 November 2021).

8. Dandona, P.; Mathieu, C.; Phillip, M.; Hansen, L.; Griffen, S.C.; Tschöpe, D.; Thorén, F.; Xu, J.; Langkilde, A.M.; Proietto, J.; et al. Efficacy and safety of dapagliflozin in patients with inadequately controlled type 1 diabetes (DEPICT-1): 24 week results from a multicentre, double-blind, phase 3, randomised controlled trial. Lancet Diabetes Endocrinol. 2017, 5, 864-876. [CrossRef]

9. Dandona, P.; Mathieu, C.; Phillip, M.; Hansen, L.; Tschöpe, D.; Thorén, F.; Xu, J.; Langkilde, A.M.; Proietto, J.; Stranks, S.; et al. Efficacy and Safety of Dapagliflozin in Patients With Inadequately Controlled Type 1 Diabetes: The DEPICT-1 52-Week Study. Diabetes Care 2018, 41, 2552-2559. [CrossRef]

10. Mathieu, C.; Dandona, P.; Gillard, P.; Senior, P.; Hasslacher, C.; Araki, E.; Lind, M.; Bain, S.C.; Jabbour, S.; Arya, N.; et al. Efficacy and Safety of Dapagliflozin in Patients With Inadequately Controlled Type 1 Diabetes (the DEPICT-2 Study): 24-Week Results From a Randomized Controlled Trial. Diabetes Care 2018, 41, 1938-1946. [CrossRef]

11. Mathieu, C.; Rudofsky, G.; Phillip, M.; Araki, E.; Lind, M.; Arya, N.; Thorén, F.; Scheerer, M.F.; Iqbal, N.; Dandona, P. Long-term efficacy and safety of dapagliflozin in patients with inadequately controlled type 1 diabetes (the DEPICT -2 study): 52-week results from a randomized controlled trial. Diabetes Obes. Metab. 2020, 22, 1516-1526. [CrossRef]

12. PRAC. SGLT2 Inhibitors: PRAC Makes Recommendations to Minimise Risk of Diabetic Ketoacidosis I European Medicines Agency. Available online: https:/ / www.ema.europa.eu/en/news/sglt2-inhibitors-prac-makes-recommendations-minimise-riskdiabetic-ketoacidosis (accessed on 3 November 2021).

13. FDA. Rejects Dapagliflozin as Treatment Add-On For Type 1 Diabetes-Diabetes. Available online: https://www.diabetes. co.uk/news/2019/jul/fda-rejects-dapagliflozin-as-treatment-add-on-for-type-1-diabetes-98751641.html (accessed on 25 November 2021). 
14. Goldenberg, R.M.; Gilbert, J.D.; Hramiak, I.M.; Woo, V.C.; Zinman, B. Sodium-glucose co-transporter inhibitors, their role in type 1 diabetes treatment and a risk mitigation strategy for preventing diabetic ketoacidosis: The STOP DKA Protocol. Diabete. Obes. Metab. 2019, 21, 2192-2202. [CrossRef]

15. Goldenberg, R.M.; Berard, L.D.; Cheng, A.Y.; Gilbert, J.D.; Verma, S.; Woo, V.C.; Yale, J.-F. SGLT2 Inhibitor-associated Diabetic Ketoacidosis: Clinical Review and Recommendations for Prevention and Diagnosis. Clin. Ther. 2016, 38, 2654-2664.e1. [CrossRef] [PubMed]

16. Burke, K.R.; Schumacher, C.; Harpe, S.E. SGLT2 Inhibitors: A Systematic Review of Diabetic Ketoacidosis and Related Risk Factors in the Primary Literature. Pharmacother. J. Hum. Pharmacol. Drug Ther. 2017, 37, 187-194. [CrossRef] [PubMed]

17. Danne, T.; Garg, S.; Peters, A.L.; Buse, J.B.; Mathieu, C.; Pettus, J.H.; Alexander, C.M.; Battelino, T.; Ampudia-Blasco, F.J.; Bode, B.W.; et al. International Consensus on Risk Management of Diabetic Ketoacidosis in Patients with Type 1 Diabetes Treated With Sodium-Glucose Cotransporter (SGLT) Inhibitors. Diabetes Care 2019, 42, 1147-1154. [CrossRef] [PubMed]

18. Qiu, H.; Novikov, A.; Vallon, V. Ketosis and diabetic ketoacidosis in response to SGLT2 inhibitors: Basic mechanisms and therapeutic perspectives. Diabetes Metab. Res. Rev. 2017, 33, e2886. [CrossRef]

19. Douros, A.; Lix, L.M.; Fralick, M.; Dell'Aniello, S.; Shah, B.R.; Ronksley, P.E.; Tremblay, É.; Hu, N.; Alessi-Severini, S.; Fisher, A.; et al. Sodium-Glucose Cotransporter-2 Inhibitors and the Risk for Diabetic Ketoacidosis: A Multicenter Cohort Study. Ann. Intern. Med. 2020, 173, 417-425. [CrossRef]

20. McGurnaghan, S.J.; on behalf of the Scottish Diabetes Research Network Epidemiology Group; Brierley, L.; Caparrotta, T.M.; McKeigue, P.M.; Blackbourn, L.A.K.; Wild, S.H.; Leese, G.P.; McCrimmon, R.J.; McKnight, J.A.; et al. The effect of dapagliflozin on glycaemic control and other cardiovascular disease risk factors in type 2 diabetes mellitus: A real-world observational study. Diabetologia 2019, 62, 621-632. [CrossRef]

21. Ueda, P.; Svanström, H.; Melbye, M.; Eliasson, B.; Svensson, A.-M.; Franzén, S.; Gudbjörnsdottir, S.; Hveem, K.; Jonasson, C.; Pasternak, B. Sodium glucose cotransporter 2 inhibitors and risk of serious adverse events: Nationwide register based cohort study. BMJ 2018, 363, k4365. [CrossRef]

22. Blau, J.E.; Tella, S.H.; Taylor, S.I.; Rother, K.I. Ketoacidosis associated with SGLT2 inhibitor treatment: Analysis of FAERS data Diabetes/Metab. Res. Rev. 2017, 33, e2924. [CrossRef]

23. Scavone, C.; Di Mauro, C.; Ruggiero, R.; Bernardi, F.F.; Trama, U.; Aiezza, M.L.; Rafaniello, C.; Capuano, A. Severe Cutaneous Adverse Drug Reactions Associated with Allopurinol: An Analysis of Spontaneous Reporting System in Southern Italy. Drugs Real World Outcomes 2020, 7, 41-51. [CrossRef]

24. Mascolo, A.; Ruggiero, R.; Sessa, M.; Scavone, C.; Sportiello, L.; Rafaniello, C.; Rossi, F.; Capuano, A. Preventable Cases of Oral Anticoagulant-Induced Bleeding: Data From the Spontaneous Reporting System. Front. Pharmacol. 2019, 10, 425. [CrossRef]

25. Di Mauro, G.; Zinzi, A.; Vitiello, F.; Restaino, M.; Sportiello, L.; Rafaniello, C.; Sullo, M.; Capuano, A. Adverse drug reactions and gender differences: What changes in drug safety? Ital. J. Gend. Specif. Med. 2019, 5, 114-122. [CrossRef]

26. Ema Summary of Product Characteristics. Available online: https://www.ema.europa.eu/en/documents/product-information/ forxiga-epar-product-information_en.pdf (accessed on 4 November 2021).

27. di Mauro, G.; Zinzi, A.; Scavone, C.; Mascolo, A.; Gaio, M.; Sportiello, L.; Ferrajolo, C.; Rafaniello, C.; Rossi, F.; Capuano, A. PCSK9 Inhibitors and Neurocognitive Adverse Drug Reactions: Analysis of Individual Case Safety Reports from the Eudravigilance Database. Drug Saf. 2021, 44, 337-349. [CrossRef] [PubMed]

28. Rafaniello, C.; Ferrajolo, C.; Sullo, M.; Gaio, M.; Zinzi, A.; Scavone, C.; Gargano, F.; Coscioni, E.; Rossi, F.; Capuano, A. Cardiac Events Potentially Associated to Remdesivir: An Analysis from the European Spontaneous Adverse Event Reporting System. Pharmaceuticals 2021, 14, 611. [CrossRef] [PubMed]

29. Mascolo, A.; Scavone, C.; Ferrajolo, C.; Rafaniello, C.; Danesi, R.; Del Re, M.; Russo, A.; Coscioni, E.; Rossi, F.; Alfano, R.; et al. Immune Checkpoint Inhibitors and Cardiotoxicity: An Analysis of Spontaneous Reports in Eudravigilance. Drug Saf. 2021, 44, 957-971. [CrossRef]

30. Sessa, M.; Rafaniello, C.; Scavone, C.; Mascolo, A.; Di Mauro, G.; Fucile, A.; Rossi, F.; Sportiello, L.; Capuano, A. Preventable statin adverse reactions and therapy discontinuation. What can we learn from the spontaneous reporting system? Expert Opin. Drug Saf. 2018, 17, 457-465. [CrossRef]

31. Scavone, C.; Di Mauro, C.; Brusco, S.; Bertini, M.; Di Mauro, G.; Rafaniello, C.; Sportiello, L.; Rossi, F.; Capuano, A. Surveillance of adverse events following immunization related to human papillomavirus vaccines: 12 years of vaccinovigilance in Southern Italy. Expert Opin. Drug Saf. 2019, 18, 427-433. [CrossRef] [PubMed]

32. Peters, A.L.; McGuire, D.K.; Danne, T.; Kushner, J.A.; Rodbard, H.W.; Dhatariya, K.; Sawhney, S.; Banks, P.; Jiang, W.; Davies, M.J.; et al. Diabetic Ketoacidosis and Related Events with Sotagliflozin Added to Insulin in Adults with Type 1 Diabetes: A Pooled Analysis of the inTandem 1 and 2 Studies. Diabetes Care 2020, 43, 2713-2720. [CrossRef]

33. Zhou, X.; Ye, X.; Guo, X.; Liu, D.; Xu, J.; Hu, F.; Zhai, Y.; Gao, Y.; Xu, X.; Dong, Z.; et al. Safety of SGLT2 Inhibitors: A Pharmacovigilance Study from 2013 to 2021 Based on FAERS. Front. Pharmacol. 2021, 12, 3618. [CrossRef]

34. Katsuhara, Y.; Ogawa, T. Acute Renal Failure, Ketoacidosis, and Urogenital Tract Infections with SGLT2 Inhibitors: Signal Detection Using a Japanese Spontaneous Reporting Database. Clin. Drug Investig. 2020, 40, 645-652. [CrossRef]

35. Colacci, M.; Fralick, J.; Odutayo, A.; Fralick, M. Sodium-Glucose Cotransporter-2 Inhibitors and Risk of Diabetic Ketoacidosis Among Adults with Type 2 Diabetes: A Systematic Review and Meta-Analysis. Can. J. Diabetes 2021, 46, 10-15.e2. [CrossRef] 
36. Taylor, S.I.; Blau, J.E.; Rother, K.I. SGLT2 Inhibitors May Predispose to Ketoacidosis. J. Clin. Endocrinol. Metab. 2015, 100, 2849-2852. [CrossRef] [PubMed]

37. McMurray, J.J.V.; Solomon, S.D.; Inzucchi, S.E.; Køber, L.; Kosiborod, M.N.; Martinez, F.A.; Ponikowski, P.; Sabatine, M.S.; Anand, I.S.; Bělohlávek, J.; et al. Dapagliflozin in Patients with Heart Failure and Reduced Ejection Fraction. N. Engl. J. Med. 2019, 381, 1995-2008. [CrossRef] [PubMed]

38. Iqbal, I.; Hamid, M.; Khan, M.A.A.; Kainat, A.; Tariq, S. Dapagliflozin-induced Late-onset Euglycemic Diabetic Ketoacidosis. Cureus 2019, 11, e6089. [CrossRef] [PubMed]

39. Pujara, S.; Ioachimescu, A. Prolonged Ketosis in a Patient with Euglycemic Diabetic Ketoacidosis Secondary to Dapagliflozin. J. Investig. Med. High Impact Case Rep. 2017, 5, 5. [CrossRef]

40. Phillip, M.; Mathieu, C.; Lind, M.; Araki, E.; di Bartolo, P.; Bergenstal, R.; Heller, S.; Hansen, L.; Scheerer, M.F.; Thoren, F.; et al. Long-term efficacy and safety of dapagliflozin in patients with inadequately controlled type 1 diabetes: Pooled 52-week outcomes from the DEPICT -1 and -2 studies. Diabete. Obes. Metab. 2021, 23, 549-560. [CrossRef]

41. Huang, Y.; Jiang, Z.; Wei, Y. Efficacy and safety of the SGLT2 inhibitor dapagliflozin in type 1 diabetes: A meta-analysis of randomized controlled trials. Exp. Ther. Med. 2021, 21, 1-11. [CrossRef]

42. Eledrisi, M.S.; Elzouki, A.N. Management of Diabetic Ketoacidosis in Adults: A Narrative Review. Saudi J. Med. Med. Sci. 2020, 8 , 165-173. [CrossRef]

43. Wang, K.M.; Isom, R.T. SGLT2 Inhibitor-Induced Euglycemic Diabetic Ketoacidosis: A Case Report. Kidney Med. 2020, 2, 218-221. [CrossRef]

44. Bader, N.; Mirza, L. Euglycemic Diabetic Ketoacidosis in a 27 year-old female patient with type-1-Diabetes treated with sodiumglucose cotransporter-2 (SGLT2) inhibitor Canagliflozin. Pak. J. Med. Sci. 2016, 32, 786-788. [CrossRef]

45. Steinmetz-Wood, S.; Gilbert, M.; Menson, K. A Case of Diabetic Ketoacidosis in a Patient on an SGLT2 Inhibitor and a Ketogenic Diet: A Critical Trio Not to Be Missed. Case Rep. Endocrinol. 2020, 2020, 1-3. [CrossRef]

46. Milder, T.Y.; Stocker, S.L.; Baysari, M.; Day, R.O.; Greenfield, J.R. Prescribing of SGLT2 inhibitors in primary care: A qualitative study of General Practitioners and Endocrinologists. Diabetes Res. Clin. Pract. 2021, 180, 109036. [CrossRef] [PubMed]

47. Vardeny, O.; Vaduganathan, M. Practical Guide to Prescribing Sodium-Glucose Cotransporter 2 Inhibitors for Cardiologists. JACC Hear. Fail. 2019, 7, 169-172. [CrossRef] [PubMed]

48. Gao, Y.; Peterson, E.; Pagidipati, N. Barriers to prescribing glucose-lowering therapies with cardiometabolic benefits. Am. Hear. J. 2020, 224, 47-53. [CrossRef] [PubMed]

49. EMA. SGLT2 Inhibitors Article 20-PRAC Assessment Report. 2016. Available online: https://www.ema.europa.eu/en/ documents/referral/sglt2-inhibitors-article-20-procedure-assessment-report_en.pdf (accessed on 21 September 2021).

50. Ruggiero, R.; Fraenza, F.; Scavone, C.; Di Mauro, G.; Piscitelli, R.; Mascolo, A.; Ferrajolo, C.; Rafaniello, C.; Sportiello, L.; Rossi, F.; et al. Immune Checkpoint Inhibitors and Immune-Related Adverse Drug Reactions: Data from Italian Pharmacovigilance Database. Front. Pharmacol. 2020, 11, 830. [CrossRef]

51. Fralick, M.; Schneeweiss, S.; Patorno, E. Risk of Diabetic Ketoacidosis after Initiation of an SGLT2 Inhibitor. N. Engl. J. Med. 2017, 376, 2300-2302. [CrossRef] 